\title{
Zentralwirksame 4-Phenylpyrane: Hydrierte Phenylchromene, Phenylaza- und Phenyloxachromene sowie Phenyl-oxa- und Phenyldioxaxanthene durch [4+2]-Cycloaddition ${ }^{1)}$
}

Fritz Eiden*, Walter Winkler, Klaus Th. Wanner und Axel Markhauser

Institut für Pharmazie und Lebensmittelchemie der Universität München, Sophienstraße 10, 8000 München 2

Eingegangen am 9. April 1984

Durch [4+2]-Cycloadditionsreaktion der Enamine 1a und 1b, 7a-7d sowie 9a und $9 \mathrm{~b}$ mit den Enonen 2a und $2 b$ sowie 11a-11c entstehen dic hydrierten und aminsubstituierten, pyrananellierten Benzol-, Pyridin-, Pyran-, Benzopyran- und Pyranopyran-Derivate 3a-3c, 8a-8d, 10, 12, 13a und 13b sowie 14a. Diese lassen sich durch Säureeinwirkung in die entsprechenden Phenylpyrane überführen; 3a 2. B. bildet so die Tetrahydrobenzopyrancarbonsäure 6.

0365-6233/85/0707-0641 5 02.5010)

- VCH Verlagsgesellschaft mbH, D-6940 Weinheim. 1985 
Centrally Acting 4-Phenylpyrans: Hydrated Phenylchromenes, Phenylaza- and Phenyloxachromenes, Phenyloxa- and Phenyldioxa-xantheses Obtained by [4+2]-Cycloaddition"

$[4+2]-$ Cycloadditions of the enamines $1 \mathrm{a}$ and $1 \mathrm{~b}, 7 \mathrm{a}-7 \mathrm{~d}$, and $9 \mathrm{a}$ and $9 \mathrm{~b}$ with the enones $2 \mathrm{a}$ and $2 \mathrm{~b}$ and 112-11c yield the hydrated and amine-substituted, pyran-fused benzene, pyridine, pyran, benzopyran and pyranupyran derivatives $3 a-3 c, 8 a-8 d, 10,12,13 a, 13 b$ and $14 a$. By treatment with acids, these compounds can be converted to the corresponding phenylpyrans. Thus, 3a for example yields the tetrahydrobenzopyrancarboxylic acid 6.

Im Zusammenhang mit Untersuchungen über Beziehungen zwischen Struktur und analgetischer Wirkung bei 4-Phenylpyran-Derivaten haben wir versucht, Verbindungen dieser Art durch [4+2]-Cycloaddition zu gewinnen. Dazu haben wir die Enamine 1a und 16 mit den gelben Oxo-phenylbutensäureestern $2 \mathrm{a}$ bzw. $2 \mathrm{~b}$ umgesetzt und in guten Ausbeuten die kristallinen, farblosen, aminsubstituierten Hexahydro-phenylchromencarbonsăureester $3 a-3 c$ erhalten.
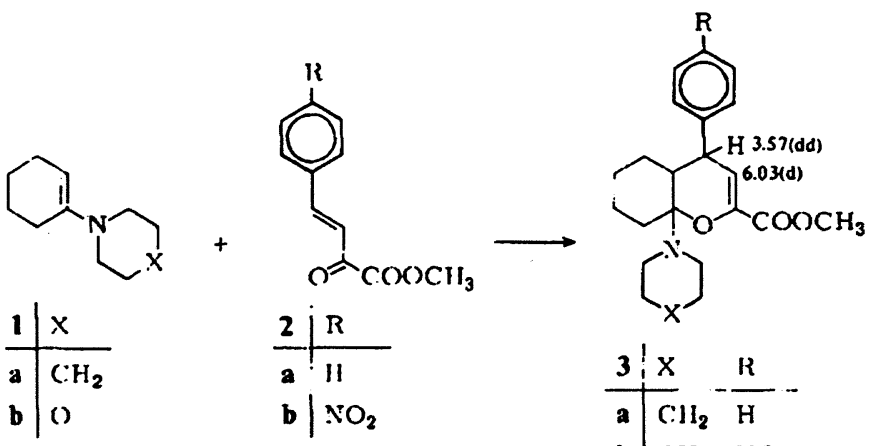

$$
\begin{array}{l|ll|l}
\text { 1 } & \mathrm{x} & \text { 2 } & \mathrm{R} \\
\hline \mathbf{a} & \mathrm{C} \mathrm{H}_{2} & \mathrm{a} & \mathrm{HI} \\
\text { b } & \mathrm{O} & \mathrm{b} & \mathrm{NO}_{2}
\end{array}
$$$$
\begin{array}{l|ll}
3 & \mathrm{X} & \mathrm{R} \\
\hline \mathbf{a} & \mathrm{C} \mathrm{H}_{2} & \mathrm{H} \\
\mathrm{b} & \mathrm{CH}_{2} & \mathrm{NO}_{2} \\
\text { c } & \mathrm{O} & \mathrm{H}
\end{array}
$$

${ }^{1} \mathrm{H}-\mathrm{N}: \mathrm{MR}-$ Daten $\delta(\mathrm{ppm})$ für $\mathbf{3 a}$

Spektren und Analysen passen gut zur angegebenen Struktur, die ${ }^{\mathbf{l}} \mathbf{H}-\mathbf{N M R}$-Signale der Protonen in 3- und 4-Stellung (s. Formel 3 und exp. Teil) schließen eine Cycloaddition in umgekehrter Richtung oder die Bildung eines Cyclobutan-Derivates aus.

Durch Erhitzen von 3a mit Salzsäure in Dioxan wurden Piperidin und Methanol abgespalten und wir erhielten $4 a$ bzw. 5a (im ' ${ }^{1} H-N M R-S p e k t r u m$ fehlt das im Spektrum von $3 a$ bei $\delta=6.03$ ppm auftretende Olefinsignal). Der entsprechende Methylester $4 b$ bzw. $5 b$ entstand aus 3a beim Erhitzen in einem Salzsäure/Methylenchlorid-Gemisch. $4 a$ ließ sich säurekatalysiert zum Tetrahydro-4H-chromen 6 dehydratisieren.

Auch mit den aus entsprechenden 4-Piperidonen darstellbaren Enaminen 7a-7d reagierte das Enon 2a unter [4+2]-Cycloaddtion, und wir erhielten die 6-Azahexahydro-phenylchromen-carbonsäureester 8a-8d in 60-70proz. Ausbeute. 


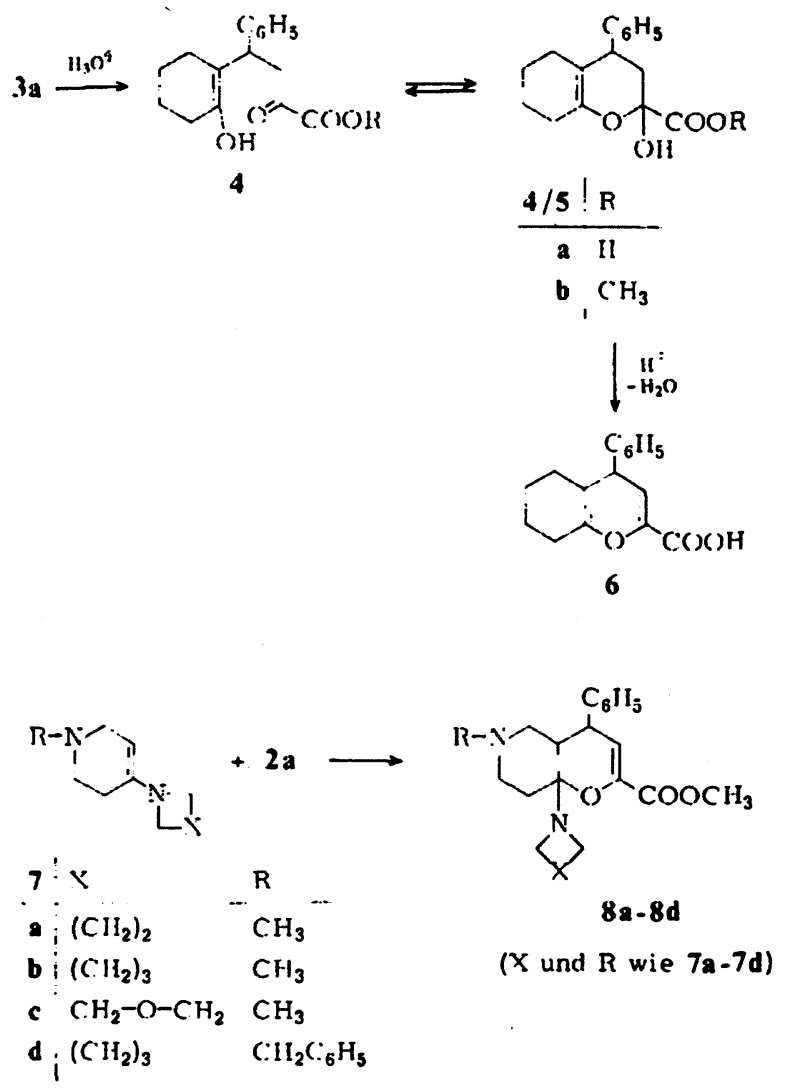

Wir haben daraufhin nach gleichem Verfahren das aus Tetrahydro-3-pyranon und Morpholin durch Erhitzen in Benzol/4-Toluolsulfonsäure dargestellte Enamin 9a (im Gemisch mit $9 \% \triangle^{3}$-Isomer ${ }^{2}$ ) mit 2a umgesetzt und (nach Reinigung) in 78proz. Ausbeute den kristallinen, farblosen, aminsubstituierten 5-Oxa-hexahydrophenylchromencarbonsäureester 10 erhalten. Die Spektren-Daten (s. Formel 10 und exp. Teil) bestätigen die Pyrano[3,2-b]pyran-Struktur.

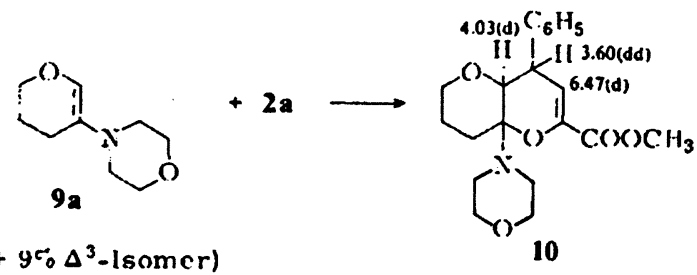

${ }^{1}$ II-NMIR-Daten $\delta$ (ppm) 
In gleicher Weise gelang auch die Cycloaddition des Enamins 1a mit dem durch Reaktion des Trimethylsilyloxy- $\Delta^{3}$-dihydropyranons mit 2-Nitrobenzaldehyd und nachfolgende Dehydratisierung erhältlichen ${ }^{3)}$ heterocyclischen Enon $11 \mathrm{a}$, und wir erhielten das hydrierte Pyrano[3,4-b]benzopyran 12.<smiles>[Y]C1OCCC2=C1OC1([Y])CCCCC1C2c1ccccc1[N+](=O)[O-]</smiles>

Schließlich konnte auch das aus Tetrahydro-3-pyranon mit Piperidin darstellbare Enamin $9 b^{3)}$ (im Gemisch mit $8 \% \Delta^{3}$-Isomer) mit dem Benzylidenpyranon 11b umgesetzt werden.

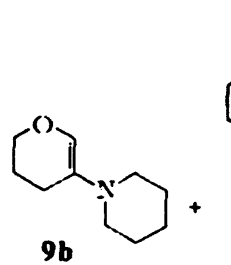

$\left(+8 m_{0} \Delta^{3}\right.$-Isomer)

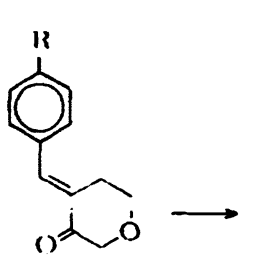

$$
\begin{array}{l|l}
11 & \mathrm{R} \\
\hline \mathrm{b} & \mathrm{NO}_{2} \\
\mathrm{c} & \mathrm{HI}
\end{array}
$$<smiles>[R]c1ccc([C@@H]2OC(N3CCCCC3)(N3CCCCC3)[C@H](OC)C3=C2COCC3)cc1</smiles>

$$
\begin{array}{l|l}
13 & \mathrm{R} \\
\hline \text { a } & \mathrm{NO})_{2} \\
\text { b } & \mathrm{H}
\end{array}
$$<smiles>[Y]c1ccc(C2=C3CCOC[C@H]3[C@@H]3CCOCC3(N3CCCCC3)O2)cc1</smiles>

$$
\begin{array}{l|l}
14 & \mathrm{R} \\
\hdashline & \mathrm{N}()_{2} \\
11
\end{array}
$$

Bei dieser Reaktion erhielten wir ein Gemisch von zwei Produkten, die sich durch fraktioniertes Kristallisieren trennen ließen. Analysen und Massenspektrum passen zu den [4+2]-Cycloadditionsprodukten 13a und 14a, sprechen also für Dipyranopyranstrukturen. Sie schließen aber die Bildung Regioisomerer oder von Cyclobutan-Derivaten nicht aus. Das gilt auch für die IR-Spektren mit Banden bei $1715 \mathrm{~cm}^{-1}$, bei denen man zwischen einer CO- oder einer Enolether-Schwingung wählen kann"; ebensowenig lassen die ${ }^{1} \mathrm{H}-\mathrm{NMR}$-Spektren eine Entscheidung zu (s. exp. Teil). Die ${ }^{13} \mathrm{C}$-Spektren von 13a sowie 14a jedoch sprechen mit den Signalen bei $\delta=99.1(5 a-C)$ bzw. 143.3 ppm $(9 a-C)$ und dem Fehlen einer $\mathrm{C}=\mathrm{O}$-Absorption für [4+2]-Cycloadditionen (s. exp. Teil).

Auch bei der Umsetzung des elektronenreicheren Benzyliden-Derivates 11c mit dem Enamin $9 b\left(+8 \% \Delta^{3}\right.$-Isomer) entstanden zwei Dipyranopyranone. Eine vollständige Trennung durch Kristallisation gelang hier nicht, das ${ }^{13} \mathrm{C}$-Spektrum von umkristalliertem 13b zeigt (mit geringer Intensität) auch die Signale von $14 \mathrm{~b}$. 
Dem Fonds der Chemischen Industrie danken wir für die finanzielle Unterstützung unserer Untersuchungen, Frau A. Kärtner für die engagicrte Mitarbeit beim Experimentieren.

\section{Experimenteller Teil}

A. Allgemeine Vorschrifi zur Darstellung der hydrierten Amino-phenyl-chromonyl-carbonsäureester (3a-3c) sowie der hydrierten Amino-phenyl-pyranopyridinylcarbonsäureester (8a-8d)

$\mathrm{Zu}$ einer Suspension von $0.01 \mathrm{~mol} 2 \mathrm{a}$ bzw. $2 b$ in $30 \mathrm{ml}$ absol. Methanol ließ man unter Kühlen (Eis/Kochsalz) und Rühren $0.015 \mathrm{~mol} 1 \mathrm{la}$ oder $1 \mathrm{~b}$ bzw. 7a-7c in $20 \mathrm{ml}$ Methanol tropfen. Es wurde bei Raumtemp. weiter gerührt, bis sich der gelbe Ester gelöst hatte (1-2 h). Dann ließ man $2 \mathrm{~h}$ bei Raumtemp. und $12 \mathrm{~h}$ bei $5^{\circ}$ stchen und saugte den farblosen Niederschlag ab. Es wurde mit kaltem Methanol und Petrolether gewaschen und, wie angegeben, umkristallisiert. Aufbewahrung unter Stickstoff und Kühlung. DC-Kontrolle: Kieselgel, Petrolether/Ethylacetat $7: 3$.

5,6,7,8-Tetrahydro-4-phenyl-8a-piperidino-4H,4aH-1-benzo-2-pyrancarbonsäuremethylester (3a): Nach Vorschrift A aus $1.90 \mathrm{~g}(0.01 \mathrm{~mol}) 2 \mathrm{and} 2.48 \mathrm{~g}(0.015 \mathrm{~mol}) 1 \mathrm{a}$. Farblose Kristalle (Methanol), Schmp. 150, Ausb. $2.80 \mathrm{~g}(79 \%) . \mathrm{C}_{22} \mathrm{H}_{29} \mathrm{NO}_{3}$ (355.5) Ber. C74.3 H 8.22 N 3.9; Gef. C74.2 H 8.28 N 3.9 Mol.-Masse 355 (ms). - IR (KBr): 3080, 3060, 3020, 2990, 2940, 2850, 2820, 1720, 1650, $1600 \mathrm{~cm}^{-1}$. - ${ }^{1} \mathrm{H}-\mathrm{NMR}\left(\mathrm{CDCl}_{3}\right): \delta(\mathrm{ppm})=7.27(\mathrm{~s}, 5 \mathrm{H}), 6.03(\mathrm{~d}, 1 \mathrm{H}, \mathrm{J}=3 \mathrm{~Hz}), 3.80(\mathrm{~s}, 3 \mathrm{H}), 3.57(\mathrm{dd}, 1 \mathrm{H}, \mathrm{J}=$ $7 \mathrm{~Hz} / 3 \mathrm{~Hz}), 2.80-2.56(\mathrm{~m}, 4 \mathrm{H}), 1.90-1.33(\mathrm{~m}, 15 \mathrm{H})$.

5,6,7,8-Tetrahydro-4-(4-nitrophenyl)-8a-piperidino-4H,4aH-1-benzo-2-pyranocarbonsäuremethylester (3b): Nach A aus $2.35 \mathrm{~g}(0.01 \mathrm{~mol}) 2 \mathrm{~b}$ und $2.48 \mathrm{~g}(0.015 \mathrm{~mol}) 1 \mathrm{a}$. Gelbliche Kristalle (Methanol), Schmp. $127^{\circ}$, Ausb. $2.20 \mathrm{~g}(55 \%)$. $\mathrm{C}_{22} \mathrm{H}_{28} \mathrm{~N}_{2} \mathrm{O}_{5}$ (400.5) Ber. C66.0 H 7.05 N 7.0; Gef. C65.6 H 7.14 N 7.1 Mol.-Masse 400 (ms). - IR (KBr): 3110, 3080, 2990, 2950, 2860, 2840, 1735, 1665, 1600, 1520, 1350 $\mathrm{cm}^{-1}$. - ${ }^{1} \mathrm{H}-\mathrm{NMR}\left(\mathrm{CDCl}_{3}\right): \delta(\mathrm{ppm})=8.20(\mathrm{~d}, 2 \mathrm{H}, \mathrm{J}=8 \mathrm{~Hz}), 7.43(\mathrm{~d}, 2 \mathrm{H}, \mathrm{J}=8 \mathrm{~Hz}), 6.08(\mathrm{~d}, 1 \mathrm{H}, \mathrm{J}=$ $3 \mathrm{~Hz}), 3.85(\mathrm{~s}, 3 \mathrm{H}), 3.47(\mathrm{dd}, 1 \mathrm{H}, \mathrm{J}=7 \mathrm{~Hz} / 3 \mathrm{~Hz}), 2.63-2.27(\mathrm{~m}, 4 \mathrm{H}), 1.83-1.47(\mathrm{~m}, 9 \mathrm{H}), 1.33-1.13(\mathrm{~m}$, $6 \mathrm{H})$.

5,6,7,8-Tetrahydro-8a-morpholino-4-phenyl-4H,4aH-1-benzo-2-pyrancarbonsäuremethylester (3c): Nach $A$ aus $1.90 \mathrm{~g}(0.01 \mathrm{~mol})$ 2and $2.51 \mathrm{~g}(0.015 \mathrm{~mol})$ 1b. Farblose Kristalle (Methanol), Schmp. $162^{\circ}$, Ausb. $2.79 \mathrm{~g}(78 \%)$. $\mathrm{C}_{21} \mathrm{H}_{27} \mathrm{NO}_{4}(357.4)$ Ber. C $70.6 \mathrm{H} 7.61 \mathrm{~N} 3.9$; Gef. C $70.6 \mathrm{H} 7.76 \mathrm{~N} 3.9$ Mol.-Masse 357 (ms). - IR (KBr): 3070, 3020, 2980, 2910, 2850, 1730, 1650, $1600 \mathrm{~cm}^{-1}$. - ${ }^{1} \mathrm{H}-\mathrm{NMR}$ $\left(\mathrm{CDCl}_{3}\right): \delta(\mathrm{ppm})=7.30(\mathrm{~s}, 5 \mathrm{H}), 6.13(\mathrm{~d}, 1 \mathrm{H}, \mathrm{J}=3 \mathrm{~Hz}), 3.87(\mathrm{~s}, 3 \mathrm{H}), 3.63(\mathrm{dd}, 1 \mathrm{H}, \mathrm{J}=7 \mathrm{~Hz} / 3 \mathrm{~Hz})$, $3.53(\mathrm{t}, 4 \mathrm{H}, \mathrm{J}=4 \mathrm{~Hz}), 2.73(\mathrm{t}, 4 \mathrm{H}, \mathrm{J}=4 \mathrm{~Hz}), 1.90-1.50(\mathrm{~m}, 9 \mathrm{H})$.

\section{3,4,5,6,7,8-Hexahydro-2-hydroxy-4-phenyl-2H-1-benzo-2-pyran-carbonsäure (5a)}

$9.24 \mathrm{~g}(0.026 \mathrm{~mol}) 3 \mathrm{a}$ wurden in $100 \mathrm{ml}$ Dioxan, $40 \mathrm{ml} 2 \mathrm{~N}-\mathrm{HCl}$ und $40 \mathrm{ml}$ Wasser 15 min rückfließend erhitzt. Dann wurde i. Vak. abgedampft, der Rūckstand in Methylenchlorid aufgenommen und mit Wasser gewaschen. Nach dem Trocknen über Magnesiumsulfat wurde eingeengt. Farblose Kristalle (Toluol/Petrolether), Schmp. 178 , Ausb. $3.9 \mathrm{~g}(55 \%) . \mathrm{C}_{16} \mathrm{H}_{18} \mathrm{O}_{4}$ (274.3) Ber. C $70.1 \mathrm{H} \mathrm{6.61;} \mathrm{Gef.} \mathrm{C}$ 70.1 H 6.62 Mol.-Masse 274 (ms). - IR (KBr): 3600-2400, 3420, 3050, 3030, 2990, 12970, 2940, 2900, $1730,1695,1635,1625,1600 \mathrm{~cm}^{-1}$. - ${ }^{1} \mathrm{H}-\mathrm{NMR}\left(\mathrm{DMSO}-\mathrm{d}_{6}\right): \delta(\mathrm{ppm})=7.46(\mathrm{~s}, 5 \mathrm{H}), 5.7-4.0(\mathrm{~m}, 1 \mathrm{H}$, $\mathrm{D}_{2} \mathrm{O}$-Austausch), 3.6-3.3 (m, $\left.1 \mathrm{H}\right), 3.3-2.8(\mathrm{~m}, 2 \mathrm{H}), 2.7-1.3(\mathrm{~m}, 8 \mathrm{H})$.

\section{3,4,5,6,7,8-Hexahydro-2-hydroxy-4-phenyl-2H-1-benzo-2-pyran-carbonsäuremethylester (5b)}

$9.24 \mathrm{~g}(0.026 \mathrm{~mol}) 3 \mathrm{a}$ wurden in $100 \mathrm{ml}$ Methylenchlorid, $30 \mathrm{ml} 2 \mathrm{~N}-\mathrm{HCl}$ und $30 \mathrm{ml}$ Wasser $4 \mathrm{~h}$ rückfließend crhitzt. Dann wurde die organische Phase abgetrennt, mit Wasser gewaschen, über 
Magnesiumsulfat getrocknet und i. Vak. eingedampft. Man erhielt gelbliches Ol, das mit Isopropanol/Diisopropylether (1:1) farblose Kristalle lieferte, Schmp. $140^{\circ}$, Ausb. $2.55 \mathrm{~g}(34 \%)$. $\mathrm{C}_{17} \mathrm{H}_{20} \mathrm{O}_{4}$ (288.3) Ber. C 70.8 H 7.00; Gef. C 70.6 H 6.89 Mol.-Masse 288 (ms). - IR (KBr): $3600-3120,3040,3030,3010,3000,2970,2940,2930,1725,1695,1660 \mathrm{~cm}^{-1}$. ${ }^{1}{ }^{1} \mathrm{H}-\mathrm{NMR}\left(\mathrm{CDCl}_{3}-\mathrm{d}_{6}\right): \delta$ $(\mathrm{ppm})=7.34(\mathrm{~s}, 5 \mathrm{H}), 3.85(\mathrm{~s}, 3 \mathrm{H}), 3.8-3.6(\mathrm{~m}, 1 \mathrm{H}), 2.8\left(\mathrm{~s}, 1 \mathrm{H}, \mathrm{D}_{2} \mathrm{O}\right.$-Austausch), 3.1-1.7 (m, 10H).

\section{5,6,7,8-Tetrahydro-4-phenyl-4H-1-benzo-2-pyrancarbonsäure (6)}

$7.13 \mathrm{~g}(0.026 \mathrm{~mol}) 5 \mathrm{a}$ wurden in $140 \mathrm{ml}$ absol. Toluol unter Zusatz von $200 \mathrm{mg}$ p-Toluolsulfonsäure 24 h am Wasserabscheider erhitzt. Dann wurde mit Wasser ausgeschüttelt und $i$. Vak. eingeengt. Beim Kühlen fielen farblose Kristalle aus. Schmp. $143^{\circ}$ (Toluol/Petrolether $1: 1$ ), Ausb. $2.93 \mathrm{~g}$ (44 \%). $\mathrm{C}_{16} \mathrm{H}_{16} \mathrm{O}_{3}$ (256.3) Ber. C 75.0 H 6.29; Gef. C 75.0 H 6.33 Mol.-Masse 256 (ms). - IR (KBr): $3500-2380,3020,2950,2910,2860,1705,1685,1645 \mathrm{~cm}^{-1} .{ }^{-} \mathrm{H}-\mathrm{NMR}$ (DMSO-d $): \delta(\mathrm{ppm})=7.48(\mathrm{~s}$, $5 \mathrm{H}), 5.98(\mathrm{~d}, 1 \mathrm{H}, \mathrm{J}=3 \mathrm{~Hz}), 4.02(\mathrm{~d}, 1 \mathrm{H}, \mathrm{J}=3 \mathrm{~Hz}), 2.3-2.0(\mathrm{~m}, 2 \mathrm{H}), 1.8-1.2(\mathrm{~m}, 6 \mathrm{H})$.

\section{B. Allgemeine Vorschrift zur Darstellung der 4-Amino-tetrahydropyridine (7a-7d)}

$\mathrm{Zu}$ einer siedenden Lösung von $0.5 \mathrm{~mol}$ Amin in $100 \mathrm{ml}$ absol. Toluol wurden unter Stickstoff und Zusatz von $200 \mathrm{mg}$ p-Toluolsulfonsäure $0.25 \mathrm{~mol}$ des 4-Piperidons in $50 \mathrm{ml}$ absol. Toluol zugetropft $(1.5 \mathrm{~h})$. Dann wurde $16 \mathrm{~h}$ rückfließend am Wasserabscheider erhitzt und i. Vak. destilliert.

1-Methyl-4-pyrrolidino-1,2,3,6-tetrahydropyridin (7a): Nach Vorschrift B aus $35.5 \mathrm{~g}(0.5 \mathrm{~mol})$ Pyrrolidin und $28.3 \mathrm{~g}$ (0.25 mol) 1-Methyl-4-piperidon, Sdp. Torr 65\%.1, Ausb. $20.1 \mathrm{~g}(50 \%)$. $\mathrm{C}_{10} \mathrm{H}_{18} \mathrm{~N}_{2}$ (166.3) Ber. C 72.2 H 10.91 N 16.9; Gef. C 71.7 H 10.82 N 17.4 Mol.-Masse 166 (ms). - IR $(\mathrm{NaCl}): 3060,2880,2830,2780,1655 \mathrm{~cm}^{-1} .{ }^{1} \mathrm{H}-\mathrm{NMR}\left(\mathrm{CDCl}_{3}\right): \delta(\mathrm{ppm})=4.27(\mathrm{t}, 1 \mathrm{H}, \mathrm{J}=3 \mathrm{~Hz})$, $3.20-3.00(\mathrm{~m}, 6 \mathrm{H}), 2.60(\mathrm{~s}, 3 \mathrm{H}, \mathrm{J}=3 \mathrm{~Hz}), 2.48(\mathrm{t}, 2 \mathrm{H}, \mathrm{J}=5 \mathrm{~Hz}), 2.43(\mathrm{~s}, 3 \mathrm{H}), 2.02-1.80(\mathrm{~m}$, 4H).

1-Methyl-4-piperidino-1,2,3,6-tetrahydropyridin (7b): Nach B aus $42.5 \mathrm{~g}(0.5 \mathrm{~mol})$ Piperidin und 28.3 $\mathrm{g}(0.25 \mathrm{~mol})$ 1-Methyl-4-piperidon, Sdp. Torr 98-100\%/6, Ausb. $29.7 \mathrm{~g}(66 \%) . \mathrm{C}_{11} \mathrm{H}_{20} \mathrm{~N}_{2}(180.3)$ Ber. C 72.3 H 11.18 N 15.5; Gef. C 72.3 H 10.99 N 15.7 Mol.-Masse 180 (ms). - IR (NaCl): 3070, 3940, $2860,2790,1655 \mathrm{~cm}^{-1}$. - ' $\mathrm{H}-\mathrm{NMR}\left(\mathrm{CDCl}_{3}\right): \delta(\mathrm{ppm})=4.58(\mathrm{t}, 1 \mathrm{H}, 3 \mathrm{~Hz}), 2.97(\mathrm{~d}, 2 \mathrm{H}, \mathrm{J}=3 \mathrm{~Hz}), 2.80$ $(\mathrm{t}, 6 \mathrm{H}, \mathrm{J}=5 \mathrm{~Hz}), 2.48(\mathrm{t}, 2 \mathrm{H}, \mathrm{J}=5 \mathrm{~Hz}), 2.37(\mathrm{~s}, 3 \mathrm{H}), 1.70-1.40(\mathrm{~m}, 6 \mathrm{H})$.

1-Methyl-4-morpholino-1,2,3,6-tetrahydropyridin (7c): Nach B aus $43.5 \mathrm{~g}(0.5 \mathrm{~mol})$ Morpholin und $28.3 \mathrm{~g}(0.25 \mathrm{~mol})$ 1-Methyl-4-piperidon, Sdp.TTorr 68-69\%.5, Ausb. 28.7 g (63 \%). $\mathrm{C}_{10} \mathrm{H}_{18} \mathrm{~N}_{2} \mathrm{O}$ (182.3) Ber. C 65.9 H 9.95 N 15.4; Gef. C65.3 H 9.80 N 15.7 Mol.-Masse 182 (ms). - IR (NaCl): 3070 , $2960,2850,2780,1660 \mathrm{~cm}^{-1} .{ }^{1} \mathrm{H}-\mathrm{NMR}\left(\mathrm{CDCl}_{3}\right): \delta(\mathrm{ppm})=4.57(\mathrm{t}, 1 \mathrm{H}, \mathrm{J}=3 \mathrm{~Hz}), 3.72(\mathrm{t}, 4 \mathrm{H}, \mathrm{J}=$ $5 \mathrm{~Hz}), 3.00(\mathrm{~d}, 2 \mathrm{H}, \mathrm{J}=3 \mathrm{~Hz}), 2.80(\mathrm{t}, 6 \mathrm{H}, \mathrm{J}=5 \mathrm{~Hz}), 2.47(\mathrm{t}, 2 \mathrm{H}, \mathrm{J}=5 \mathrm{~Hz}), 2.33(\mathrm{~s}, 3 \mathrm{H})$.

1-Benzyl-4-piperidino-1,2,3,6-tetrahydropyridin (7d): Nach Vorschrift B aus $42.5 \mathrm{~g}$ (0.5 mol) Piperidin und $47.2 \mathrm{~g}(0.25 \mathrm{~mol})$ 1-Benzyl-4-piperidon. Nach dem Abdestillieren des Lösungsmittels wurde das Enamin ohne weitere Reinigung mit $2 a$ umgesetzt.

4,4a,5,6,7,8-Hexahydro-6-methyl-4-phenyl-8a-pyrrolidino-2-8aH-pyrano[3,2-c]pyridincarbonsäuremethylester (8a): Nach $A$ aus $1.9 \mathrm{~g}(0.01 \mathrm{~mol}) 2 \mathrm{a}$ und $2.50 \mathrm{~g}(0.015 \mathrm{~mol}) 7 \mathrm{a}$. Farblose Kristalle (Methanol), Schmp. 142 , Ausb. $2.06 \mathrm{~g}(58 \%) . \mathrm{C}_{21} \mathrm{H}_{28} \mathrm{~N}_{2} \mathrm{O}_{3}$ (356.5). Ber. C 70.8 H 7.92 N 7.9; Gef. C 70.4 H 8.22 N 8.1 Mol.-Masse 356 (ms). - IR (KBr): 3050, 2960, 2830, 1730, 1660, $1610 \mathrm{~cm}^{-1}$. ${ }^{1} \mathrm{H}-\mathrm{NMR}\left(\mathrm{CDCl}_{3}\right): \delta(\mathrm{ppm})=7.25(\mathrm{~s}, 5 \mathrm{H}), 6.05(\mathrm{~d}, 1 \mathrm{H}, \mathrm{J}=3 \mathrm{~Hz}), 3.81(\mathrm{~s}, 3 \mathrm{H}), 3.55(\mathrm{dd}, 1 \mathrm{H}, \mathrm{J}=$ $7 \mathrm{~Hz} / 3 \mathrm{~Hz}), 2.67(\mathrm{t}, 4 \mathrm{H}, \mathrm{J}=5 \mathrm{~Hz}), 2.25(\mathrm{~s}, 3 \mathrm{H}), 2.36-2.10(\mathrm{~m}, 4 \mathrm{H}), 1.85-1.65(\mathrm{~m}, 7 \mathrm{H})$.

4,4a,5,6,7,8-Hexahydro-6-methyl-4-phenyl-8a-piperidino-2-8aH-pyrano/3,2-clpyridin-carbonsäuremethylester (8b): Nach A aus $1.9 \mathrm{~g}(0.01 \mathrm{~mol}) 2 \mathrm{and} 2.70 \mathrm{~g}(0.015 \mathrm{~mol}) 7 \mathrm{~b}$. Farblose Kristalle 
(Methanol). Schmp. 116 . Ausb. $2.07 \mathrm{~g}\left(56 \%\right.$ ). $\mathrm{C}_{22} \mathrm{H}_{30} \mathrm{~N}_{2} \mathrm{O}_{3}$ (370.5) Ber. C 71.3 H 8.16 N 7.6; Gef. C 70.9 H 8.32 N $7.3 \mathrm{Mol} .-$ Masse 370 (ms). - IR (KBr): 3060, 2940, 2820, 1730, 1655, $1600 \mathrm{~cm}^{-1}$. ${ }^{\prime} \mathrm{H}-\mathrm{NMR}\left(\mathrm{CDCl}_{3}\right): \delta(\mathrm{ppm})=7.23(\mathrm{~s}, 5 \mathrm{H}) .6 .03(\mathrm{~d}, 1 \mathrm{H}, \mathrm{J}=3 \mathrm{~Hz}) .3 .77(\mathrm{~s}, 3 \mathrm{H}), 3.52(\mathrm{dd}, 1 \mathrm{H}, \mathrm{J}=$ $7 \mathrm{~Hz} / 3 \mathrm{~Hz}), 2.70(\mathrm{t} .4 \mathrm{H} . \mathrm{J}=5 \mathrm{~Hz}), 2.20(\mathrm{~s}, 3 \mathrm{H}), 2.33-2.07(\mathrm{~m}, 4 \mathrm{H}), 1.80-1.50(\mathrm{~m}, 9 \mathrm{H})$.

4.4a,5,6,7,8-Hexahydro-6-methyl-8a-morpholino-4-phenyl-2-8aH-pyrano/3,2-c/pyridincarbonsäuremethylester (8c): . Nach $A$ aus $1.9 \mathrm{~g}(0.01 \mathrm{~mol}) 2 \mathrm{a}$ und $2.73 \mathrm{~g}(0.015 \mathrm{~mol}) .7 \mathrm{c}$. Farblose Kristalle (Methanol). Schmp. 104 , Ausb. $2.42 \mathrm{~g}(65 \%)$ ). $\mathrm{C}_{21} \mathrm{H}_{28} \mathrm{~N}_{2} \mathrm{O}_{4}$ (327.5) Ber. C67.7 H 7.58 N 7.5; Gef. C 67.8 H 7.60 N 7.6 Mol.-Masse 372 (ms). - IR (KBr): $3030,2980,2850.2810,1730,1660,1610 \mathrm{~cm}^{-1}$. ${ }^{\prime} \mathrm{H}-\mathrm{NMR}\left(\mathrm{CDCl}_{3}\right): \delta(\mathrm{ppm})=7.28(\mathrm{~s}, 5 \mathrm{H}), 6.23(\mathrm{~d}, 1 \mathrm{H}, 3 \mathrm{~Hz}), 3.87$ (s, $\left.3 \mathrm{H}\right), 3.60$ (dd, $1 \mathrm{H}, \mathrm{J}=$ $7 \mathrm{~Hz} \cdot 3 \mathrm{~Hz}), 3.45(\mathrm{t} .4 \mathrm{H}, \mathrm{J}=5 \mathrm{~Hz}), 2.58(\mathrm{t} .8 \mathrm{H} . \mathrm{J}=5 \mathrm{~Hz}), 2.37(\mathrm{~s}, 3 \mathrm{H}), 2.15-2.00(\mathrm{~m}, 3 \mathrm{H})$.

6-Benzyl-4,4a,5,6,7,8,8a-hexahydro-4-phenyl-4-piperidino-2-8aH-pyrano/3,2-c/pyridincarbonsäuremethylester (8d): Nach $A$ aus $1.9 \mathrm{~g}(0.01 \mathrm{~mol}) 2 \mathrm{a}$ und $3.85 \mathrm{~g}(0.015 \mathrm{~mol}) 7 \mathrm{~d}$. Farhlose Kristallc (Methanol). Schmp. $111^{\circ}$. Ausb. $3.13 \mathrm{~g}(70 \%)$. $\mathrm{C}_{28} \mathrm{H}_{34} \mathrm{~N}_{2} \mathrm{O}_{3}$ (446.6) Ber. C 75.3 H 7.67 N 6.3; Gef. C 75.0 H 7.60 N 6.3 Mol.--Masse 446 (ms). - IR (KBr): 3030, 3010, 2995, 2965. 2925, 2910, 2885, 1715 , $1650 \mathrm{~cm}^{-1}$. - ' $\mathrm{H} \cdot \mathrm{NMR}\left(\mathrm{CDCl}_{3}-\mathrm{d}_{6}\right): \delta(\mathrm{ppm})=7.31(\mathrm{~s}, 5 \mathrm{H}), 7.09(\mathrm{~s}, 5 \mathrm{H}), 5.99(\mathrm{~d}, 1 \mathrm{H}, \mathrm{J}=3 \mathrm{~Hz})$, $4.0-3.8$ (m, IH), 3.74 (s. $3 \mathrm{H}), 3.40$ (s. $2 \mathrm{H}), 2.8-2.2(\mathrm{~m}, 8 \mathrm{H}), 2.2-1.8(\mathrm{~m}, 3 \mathrm{H}), 1.37$ (s, 6H).

\section{4a,6,7,8,8a-Hexahydro-8a-morpholino-4-phenyl-2-pyrano[3,2-b]pyrancarbonsäuremethylester} (10)

$\mathrm{Zu}$ ciner Suspension von $1.9 \mathrm{~g}(0.01 \mathrm{~mol}) 2 \mathrm{a}$ in $25 \mathrm{~mol}$ absol. Isopropanol wurden unter Kühlung (Eiskochsalz-Gemisch) unter Rühren $3.38 \mathrm{~g}(0.02 \mathrm{~mol}) 9 \mathrm{a}^{3}$ ) in $25 \mathrm{ml}$ Isopropanol zugetropft (ctwa 30 min). Dann wurde unter Kühlung gerührt, bis sich der gelbe Ester gelöst hatte (etwa $3 \mathrm{~h}$ ). Die Umsctzung wurde de kontrolliert (Kieselgel/Toluol/Ethylacetat $1: 1$ ). Nach $15 \mathrm{~h}$ bei $5^{\circ}$ wurde der farblose Niederschlag aus Petrolether umkristallisicrt. Schmp. 134-135 . Ausb. 2.82 g (78 \%). $\mathrm{C}_{201} \mathrm{H}_{2 \varsigma} \mathrm{NO}_{5}$ (359.4) Ber. C $66.8 \mathrm{H} 7.01$ N 3.9; Gef. C 66.7 H 7.00 N 3.5 Mol.-Masse 359 (ms). - IR (KBr): $3090.3030,2960,2880,2840,2820,1725,1675,1610 \mathrm{~cm}^{-1}$. - 'H-NMR $\left(\mathrm{CDCl}_{3}\right): \delta(\mathrm{ppm})=$ 7.33 (s. $5 \mathrm{H}) .6 .47$ (d. $1 \mathrm{H}, \mathrm{J}=3 \mathrm{~Hz}), 4.03(\mathrm{~d}, 1 \mathrm{H}, \mathrm{J}=1 \mathrm{~Hz}), 3.83(\mathrm{~s}, 3 \mathrm{H}), 3.60(\mathrm{dd}, 1 \mathrm{H}, \mathrm{J}=3 \mathrm{~Hz} / 1 \mathrm{~Hz})$, $3.43(\mathrm{t} .2 \mathrm{H} . \mathrm{J}=5 \mathrm{~Hz}) .3 .20-3.00(\mathrm{~m}, 4 \mathrm{H}) .2 .83-2.33(\mathrm{~m}, 4 \mathrm{H}), 2.27-1.60(\mathrm{~m}, 4 \mathrm{H})$.

1-13.4.8,9.10,10a-Hexahydro-10-(4-nitrophenyl)-4a-(2H.6H,dipyrano/3,2-b:4',3'-e/pyranyl))/piperidin (13a) und 1-13,4,5,5a,6,7-Hexahydro-5-14-nitrophenyl)-9a-(1H,9H,dipyranol3,4-b:4',3'. elpyranyl)]-piperidin (14a)

Eine Lösung von $0.46 \mathrm{~g}(2 \mathrm{mmol}) 11 \mathrm{~b}^{3)}$ und $0.69 \mathrm{~g}$ (4 mmol).9b") in $2 \mathrm{ml}$ absol. Benzol wurde $24 \mathrm{~h}$ bei 50-60 (Badtemp.) gerührt. Es wurde i. Vak. abgedampft und der Rückstand aus absol. Methanol umkristallisiert. Blaßgelbe Kristalle. Schmp. 145-149., Ausb. 0.5: \& $64 \%$ ). $\Upsilon_{22} \mathrm{H}_{28} \mathrm{~N}_{2} \mathrm{O}_{5}(400.5)$ Ber. C 66.0 H 7.05 N 7.0: Gef. C65.9 H 7.10 N 7.0 Mol.-Masse 400 (ms). - IR (KBr): 2930, 1715,1510 $\mathrm{cm}^{-1}$. - ' $\mathrm{H}-\mathrm{NMR}\left(\mathrm{CDCl}_{3}\right): \delta(\mathrm{ppm})=0.52-1.33(\mathrm{~m}, 6 \mathrm{H}), 1.33-2.70(\mathrm{~m}, 10 \mathrm{H}), 3.07-3.60(\mathrm{~m}, 2 \mathrm{H})$, $3.68-4.20(\mathrm{~m} .6 \mathrm{H}) .7 .45(\mathrm{~d} . \mathrm{J}=9 \mathrm{~Hz}, 2 \mathrm{H}), 8.10(\mathrm{~d}, \mathrm{~J}=9 \mathrm{~Hz}, 2 \mathrm{H}) .-{ }^{13} \mathrm{C}-\mathrm{NMR}\left(\mathrm{CDCl}_{3}\right): \delta(\mathrm{ppm})=22.8$ $(t) .24 .2(t) .24 .5 .24 .7(t), 26.6(t) .46 .2(t), 47.2(d) .64 .4(t) .64 .9(t), 67.6(t), 76.5(d), 86.9(s), 99.1$ (s). 123.0 (d). 130.7 (d). 143.3 (s). 146.1 (s). 148.4 (s).

Die beim Umkristallisieren von 13a crhaltene Mutterlauge wurde auf $-5^{\circ}$ gckühlt. 14a fiel als hellbrauner, durchscheinender Kristall aus. Schmp. 139.5-140, Ausb. $0.10 \mathrm{~g}(13 \%)$. $\mathrm{C}_{22} \mathrm{H}_{28} \mathrm{~N}_{2} \mathrm{O}_{5}$ (400.5) Ber. C 66.0 H 7.05 N 7.0; Gef. C 66.2 H 7.11 N 7.0 Mol.-Masse 400 (ms). - IR (KBr): 2940, $1710.1510 \mathrm{~cm}^{-1}$. - $\mathrm{H}-\mathrm{NMR}\left(\mathrm{CDCl}_{3}\right): \delta(\mathrm{ppm})=0.87-1.38(\mathrm{~m}, 6 \mathrm{H}) .1 .52-2.83(\mathrm{~m} .9 \mathrm{H}), 3.0-3.23(\mathrm{~m}$, 1H). 3.32-3.97 (m. 6H). 3.97-4.20 (m. 2H). 7.37 (d. J $=9 \mathrm{~Hz} .2 \mathrm{H}), 8.12(\mathrm{~d} . \mathrm{J}=9 \mathrm{~Hz}, 2 \mathrm{H}) .{ }^{13} \mathrm{C} . \mathrm{NMK}$ $\left(\mathrm{CDCl}_{3}\right): \delta(\mathrm{ppm})=24.7 .25 .6,26.7(\mathrm{t}), 29.8(\mathrm{t}), 38.6(\mathrm{~d}), 45.8(\mathrm{~d}), 45.9(\mathrm{t}), 64.5,65.1,65.7,65.8,87.5$ (s). 99.2 (s). 123.3 (d). 130.1 (d). 144.3 (s), 146.4 (s). 150.9 (s). 
1-[3,4,5,5a,6,7,8,9-Octahydro-5-(2-nitrophenyl)-9a-(1H-pyrano[3,4-b]-1-benzopyranol)]-piperidin (12)

Darstellung wie bei $13 \mathrm{~b}$ aus $0.46 \mathrm{~g}(2.0 \mathrm{mmol}) 11 \mathrm{a}^{3)}$ und $0.66 \mathrm{~g}(4.0 \mathrm{mmol}) 1 \mathrm{a}$ in $2 \mathrm{ml}$ absol. Benzol bei $80^{\circ}$ (Badtemp.), Reaktionsdauer $12 \mathrm{~h}$. Blaßgelbe Kristalle (Methanol), Schmp. 150-151 ${ }^{\circ}$, (Zers.), Ausb. $0.39 \mathrm{~g} \mathrm{(49 \% ).} \mathrm{C}_{23} \mathrm{H}_{30} \mathrm{~N}_{2} \mathrm{O}_{4}$ (398.5) Ber. C $69.3 \mathrm{H} 7.59 \mathrm{~N} 7.0$; Gef. C $68.2 \mathrm{H} 7.65 \mathrm{~N} 6.6$ Mol.-Masse 398 (ms). - IR (KBr): 2930, 1700, $1518 \mathrm{~cm}^{-1}$. - ${ }^{1} \mathrm{H}-\mathrm{NMR}\left(\mathrm{CDCl}_{3}\right): \delta(\mathrm{ppm})=0.88-2.97$ (m, 21H), 3.43-3.95 (m, 3H), 3.95-4.23 (m, 2H), 7.12-7.93 (m, 4H).

\section{$1-\left\{3,4,8,9,10,10 a-H e x a h y d r o-10-\right.$ phenyl-4a- $\left(2 H, 6 H\right.$-dipyrano $\left.\left./ 3,2-b: 4^{\prime}, 3^{\prime}-e / p y r a n y l\right)\right]-$ piperidin} (13b)

Eine Mischung aus $0.30_{\mathrm{g}}(1.58 \mathrm{mmol}) 11 \mathrm{c}^{3)}, 0.40 \mathrm{~g}(2.37 \mathrm{mmol}) \mathrm{gb}^{3)}$ und $3.5 \mathrm{ml}$ absol. Methanol wurde $16 \mathrm{~h}$ bei $45^{\circ}$ (Badtemp.) gerührt. Dann wurde mit Methanol verdürnt und nach Zusatz von Aktivkohle filtriert. Bei Raumtemp. farblose Kristalle, Schmp. 107-111 ${ }^{\circ}$, Ausb. 0.18 g (32 \%). $\mathrm{C}_{22} \mathrm{H}_{29} \mathrm{NO}_{3}$ (355.5) Ber. C 74.3 H 8.22 N 3.9; Gef. C 74.4 H 8.20 N 4.0 Mol.-Masse 355 (ms). - IR (KBr): 1705, $1490 \mathrm{~cm}^{-1}$. - 'H-NMR $\left(\mathrm{CDCl}_{3}\right): \delta(\mathrm{ppm})=0.77-2.78(\mathrm{~m}, 16 \mathrm{H}), 3.13-3.52(\mathrm{~m}, 2 \mathrm{H})$, 3.52-4.23 (m, 6H), 7.07-7.50 (m, 5H). $-{ }^{13} \mathrm{C}-\mathrm{NMR}\left(\mathrm{CDCl}_{3}\right): \delta(\mathrm{ppm})=23.1(\mathrm{t}), 24.3,25.0,26.7(\mathrm{t})$, $46.3(t), 47.1(\mathrm{~d}), 64.6(\mathrm{t}), 65.2(\mathrm{t}), 67.5(\mathrm{t}), 77.4(\mathrm{~d}), 87.0(\mathrm{~s}), 100.6(\mathrm{~s}), 125.7$ (d), $127.8(\mathrm{~d}), 129.9$ (d), $140.3(\mathrm{~s}), 142.4(\mathrm{~s})$.

\section{Literatur}

1 109. Mitt. über Untersuchungen an Pyran-Derivaten; 108. Mitt. F. Eiden und K. Th. Wanner, Arch. Pharm. (Weinheim) 318, 548 (1985).

2 F. Eiden und K. Th. Wanner, Arch. Pharm. (Weinheim) 317, 958 (1984).

3 F. Eiden und K. Th. Wanner, Liebigs Ann. Chem. 1984, 1759.

4 H. Volkmann, Handbuch der Infrarotspektroskopie, S. 336, Verlag Chemie, Weinheim 1972. 